\title{
Autologous PBLs Retrovirally-transduced with TCRs Targeting Neoantigens
}

National Cancer Institute

\section{Source}

National Cancer Institute. Autologous PBLS Retrovirally-transduced with TCRS Targeting

Neoantigens. NCI Thesaurus. Code C146937.

Autologous human peripheral blood lymphocytes (PBLS) transduced with a retroviral vector encoding T-cell receptors (TCRs) specific for a patient's individual and unique mutated antigens, with potential immunostimulating and antineoplastic activities. Tumor cells are analyzed to identify and isolate specific mutated tumor-associated antigens (TAAs) that are expressed by the patient's tumor cells; then T-cell receptor coding sequences are engineered to targ et the patient's TAAs and inserted into retroviral vectors. After transduction, expansion in culture, and reintroduction into the patient, neoantigen-specific TCRs retroviral vector-transduced autologous PBLs recognize and bind to tumor cells expressing the patient's neoantigens, which results in a specific cytotoxic T-lymphocyte (CTL)-mediated immune response against the patient's tumor cells. 